\title{
Multidisciplinary Teams and Clinics: Better Care or Just More Care
}

\author{
Martin A. Makary, MD, MPH \\ Departments of Surgery and Health Policy and Management, Johns Hopkins University School of Medicine, Baltimore, \\ MD
}

As knowledge increases, so does the complexity of the healthcare system. The result has been that patients are often ping-ponged between multiple specialists for a single problem. In the case of cancer care, such shuttling to different doctors' offices can mean unnecessary delays at a period when time is critical. These delays are preventable. Delays should not occur simply because specialists live in separate silos and have separate schedules, offices, and availability. Such fragmented care also results in lower patient satisfaction and creates more opportunities for patients to fall through the cracks.

Multidisciplinary teams tackle many of these problems, making an increasingly complex and fragmented healthcare system more user-friendly for the patient. Using a onestop shopping approach, patients can receive input from a multispecialty group, including providers such as a medical and surgical oncologist, radiologist, social worker, genetic counselor, nutritionist, and even a counselor. These teams provide a joint team decision which is often reached during a single patient visit so that treatment can begin immediately. It also provides patients with the collective wisdom of a group of experts. At Johns Hopkins, I now steer most of my new patients into one of two multidisciplinary clinics I staff, each with a unique group of disease-specific specialists: a multidisciplinary pancreatic cancer clinic and a separate multidisciplinary pancreatitis clinic. In each of these two clinics, patients receive disease-specific medical

An Editoral for: "Quality of care management decisions by multidisciplinary cancer teams: a systematic review" by Benjamin Lamb et al. (ASO-2011-01-0117).

(C) Society of Surgical Oncology 2011

Published Online: 11 May 2011

M. A. Makary, MD, MPH

e-mail: mmakary1@jhmi.edu advice and education, rather than a series of independent, separately scheduled opinions each qualified with the disclaimer to see what the other specialist thinks. Moreover, patient safety is improved when all the treatment considerations are discussed among the team, rather than relying on multiple transmissions of medical communications between providers (letters, notes, and records). ${ }^{1}$

Lamb et al. nicely point out the growing trend towards multidisciplinary teams in healthcare and the benefits of these teams for patient care. ${ }^{2}$ They point out that up to half of patients will receive a treatment recommendation change from a prior treatment plan as a result of the team approach. This finding demonstrates the wide variations in practice patterns and the collective power of an expert group. The future of medicine will likely represent a division of labor not by historical department (i.e., surgery, medicine, radiology) but rather by disease process (gastroesophageal reflux, lung cancer, etc.). Already, some hospitals have begun to restructure their care delivery to create heart centers composed of cardiologists and cardiac surgeons working in the same department. Gastroenterology surgery/medicine and plastic surgery/dermatology are other fields growing closer to being a unified department within a hospital. Cancer care represents the fastest growing area of consolidation given its multidisciplinary nature. Clearly, the future of medical care is in multidisciplinary teams, and many hospitals have already begun to adopt this approach.

While multidisciplinary teams make sense and are appealing to many providers and patients, one flag that has been raised about these teams is cost. Policy experts have noted that patients are more likely to have higher utilization when they are seen by a group of specialists. For example, policy-makers have observed that a patient is more likely to have radiotherapy when a radiation therapist is routinely involved in the care of every patient - a good 
trend when radiotherapy is indicated, but a dangerous trend when it is not. The same may be true for any specialist involved in the team. While more care may be better care, standards need to be used to guide treatment decisions in a measurable way when appropriate. Many doctors involved in such teams acknowledge that it is more difficult to avoid a nonindicated consultation when the consultant is in the multidisciplinary conference and requests to see the patient. Moreover, when someone on the team suggests "you could get a [given test]," the lack of utility of the test can sometimes be ignored rather than addressed. These issues need to be addressed by the leader of the multidisciplinary team with standards and national and local guidelines to prevent overutilization by these teams. Evaluating suggestions in light of current evidence and its overall impact on patient management needs to be paramount for teams to improve care and not worsen it. Failure to manage utilization based on appropriateness in this way could result in policy-makers scrutinizing such multiprovider clinics on cost and not on quality of care. Multidisciplinary teams can result in lower or higher costs; they can diagnose critical problems earlier and intervene before further complications develop, or they can add tests, consultations, and treatments that are not evidence-based and are of little benefit to the patient's condition. The resultant quality and cost benefit to the patient and the system depend on the way in which these teams are managed and led. Every effort should be made to improve efficiency and provide optimal care through the wisdom of a large team, while being vigilant to avoid overutilization that does not have an associated patient benefit.

The field of quality and safety in medical care has historically suffered from having poor-quality science. The result has been a series of new innovations that do not have measureable results to compare with standard care. Lamb et al. report that the scientific quality of studies on multidisciplinary teams is "low to medium," meaning that better methods are needed to establish the benefit of such a common-sense team approach. Lamb et al. have advanced the science of quality care by objectively evaluating the impact of these clinics. The field of quality of care and health services research desperately needs to have the same scientifically sound methods to evaluate innovation as those that we use to evaluate the benefit and safety of new chemotherapeutic agents.

\section{REFERENCES}

1. Makary MA, Sexton JB, Freischlag JA, Millman EA, Pryor D, Holzmueller C, et al. Patient safety in surgery. Ann Surg. 2006;243(5);628-35.

2. Lamb BW, Brown KF, Nagpal K, Vincent C, Green JSA, Sevdalis N. Quality of care management decisions by multidisciplinary cancer teams: a systematic review. Ann Surg Oncol. 2011. 